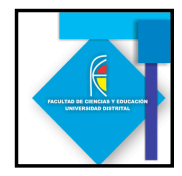

\title{
ANÁLISIS DE PRECONCEPTOS SOBRE VELOCIDAD Y RAPIDEZ EN ESTUDIANTES DE PRIMER SEMESTRE DE LICENCIATURA EN FÍSICA, UNIVERSIDAD DISTRITAL FRANCISCO JOSÉ DE CALDAS.
}

\section{ANALYSIS PRECONCEPTIONS ABOUT SPEED AND FAST IN FIRST SEMESTER STUDENTS OF DEGREE IN PHYSICS, UNIVERSIDAD DISTRITAL FRANCISCO JOSE DE CALDAS.}

\author{
Andres Fernando Pedraza ${ }^{1}$ \\ Malory Johana Sánchez ${ }^{2}$
}

\begin{abstract}
Resumen
Las estructuras mentales preconcebidas en que el alumno articula el mundo son de vital importancia en la elaboración de un nuevo aparato cognitivo. De esta manera, se busca a partir de dos conceptos básicos en el área de cinemática realizar un estudio sobre las ideas previas de los estudiantes de primer semestre pertenecientes a la carrera de licenciatura en física de la Universidad Distrital Francisco José de Caldas por medio de el uso en el aula de dos simulaciones computarizadas guiadas, con el fin de diagnosticar la clase de conceptualización que tenga el grupo.
\end{abstract}

Palabras clave: Ideas previas, cinemática, simulación computarizada.

\begin{abstract}
Preconceived mental structures in which the student articulates the world are of vital importance in the development of a new cognitive device. Thus, it seeks from two basic concepts in the fields of kinematics realize a study of two basic concepts in the area of kinematics on previous ideas in the first semester students belonging to the career degree in physics from the Universidad Distrital Francisco José de Caldas through the classroom use computer simulations of two tours, in order to diagnose the kind of conceptualization that has the group.
\end{abstract}

Keywords: Previous ideas, kinematics, computer simulation.

\section{Actividad}

\footnotetext{
${ }^{1}{ }^{1}$ Universidad Distrital Francisco José de Caldas. Grupo de investigación FISINFOR Semillero de investigación SILAB afpedrazah@correo.udistrital.edu.co.

${ }^{2}$ Universidad Distrital Francisco José de Caldas. Grupo de investigación FISINFOR Semillero de investigación SILAB , mjsanchezb@correo.udistrital.edu.co
} 


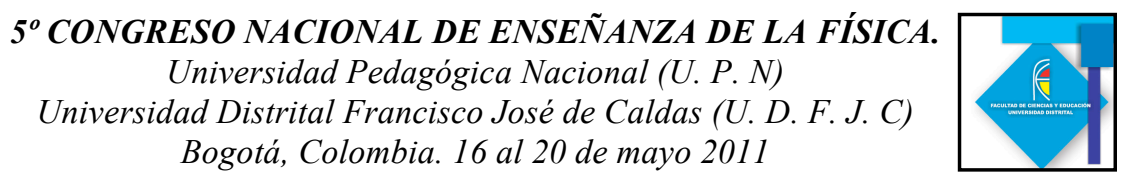

El día 23 de abril de 2010 en los laboratorios de la física de la Universidad Distrital Francisco José de Caldas a un grupo de 25 estudiantes de primer semestre pertenecientes a la asignatura física y matemática básicas, se les aplico una encuesta semiestructurada anónima con 5 preguntas en relación a una problemática simulada en el área de cinemática con la plataforma Easy Java Simulations@ facilitando que el alumno relacione diferentes tipos de representación, favoreciendo la comprensión de teorías y modelos (Sierra,2002) con el fin de poder generar un cambio conceptual por medio de la simulación y encaminar de manera adecuada las preguntas generadas que se puedan presentar por parte de los estudiantes abordando diferentes grados de contextualización de una determinada variable física dependiendo del contexto dentro del cual se están haciendo referencia a ella, (Hurtado y Lombana 2006)

El fin es crear una mesa de discusión, en la cual los estudiantes por interés propio (dado que no debía tomarse como una obligación) podían participar generando opiniones divergentes y controversia sobre las distintas preconcepciones que se pudieran presentar a la hora de poder manifestar sus interrogantes; de la misma manera se quiere presentar de manera adicional a los estudiantes las limitaciones que posee el modelo de acuerdo a las condiciones que puede tener cualquier fenómeno físico. En cuanto a la base de las discusiones realizadas con los estudiantes se tomo la encuesta realizada el mismo día de la confrontación de preconceptos, dado que resultó interesante observar como iban evolucionando los conceptos de un estudiante a otro (aunque la palabra evolución va dada por el concepto científico más aceptado actualmente, y no por el mejor concepto ya que eso es mas subjetivo), por esta razón después de la aplicación del software interactivo se vuelve a realizar la aplicación de la misma encuesta con el fin de poder analizar punto a punto de la misma como ocurrió en algunos estudiantes dicho cambio conceptual.

A continuación se mostrará el programa empleado, y los resultados de la aplicación de la encuesta al grupo seleccionado.

\section{El programa y las simulaciones}

La simulación se realizó en el programa Easy Java Simulations $®$ (Esquembre, 2005) y se planteaban las siguientes problemáticas:

\section{Simulación 1}

Dos esferas partían de un mismo punto con una misma velocidad inicial en trayectoria recta, una seguía una trayectoria horizontal (esfera azul) mientras la otra tenia una inclinación vertical (esfera roja), luego en un tiempo dado se detenía la simulación. Se iniciaba la sesión preguntando cual de las dos esferas había recorrido mas distancia, posteriormente los estudiantes daban sus respuestas y sus argumentos.

\section{Simulación 2}

Se presentaban nuevamente 2 esferas partiendo de un mismo punto, esta vez una seguía una trayectoria rectilínea horizontal, mientras la otra seguía una trayectoria parabólica, cuando la esfera azul llegaba a un punto cero se detenía la simulación y nuevamente se realizaba una serie de interrogantes acerca de el problema, finalmente, se realiza la 
$5^{\circ}$ CONGRESO NACIONAL DE ENSEÑANZA DE LA FÍSICA.

Universidad Pedagógica Nacional (U. P. N)

Universidad Distrital Francisco José de Caldas (U. D. F. J. C)

Bogotá, Colombia. 16 al 20 de mayo 2011

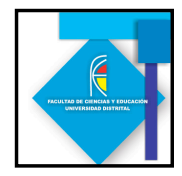

encuesta antes y después de realizar la respectiva explicación de los fenómenos observados.

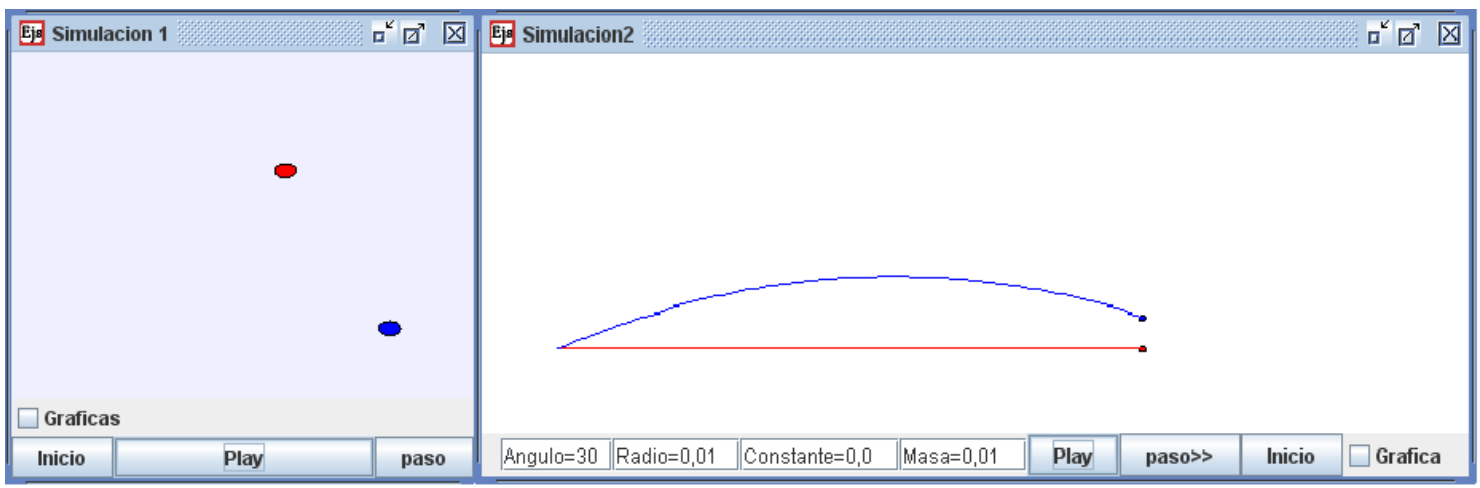

Figura 1: Simulaciones realizadas

\section{Encuesta}

A la primera pregunta: ¿Que entiende usted por velocidad? los estudiantes en la encuesta contestaron:

Distancia sobre tiempo

Relacion distancia tiempo en una direccion d

Otros

NS/NR

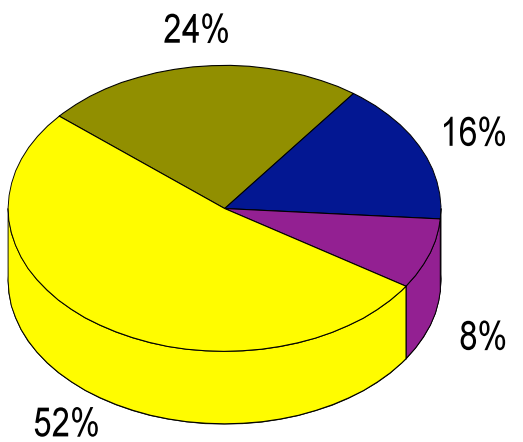

Distancia sobre tiempo

Relacion distancia tiempo en una direccion dac Otros

NS/NR

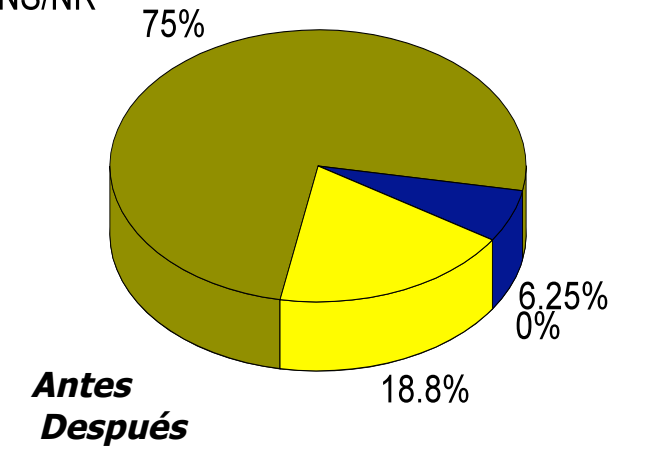


A la segunda pregunta: ¿Que lectura proporciona el velocímetro de un automóvil? Respondieron:

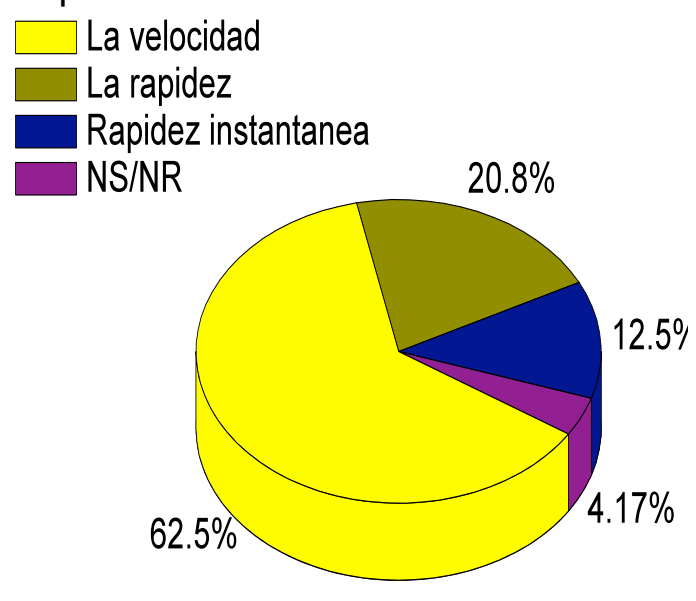

Antes

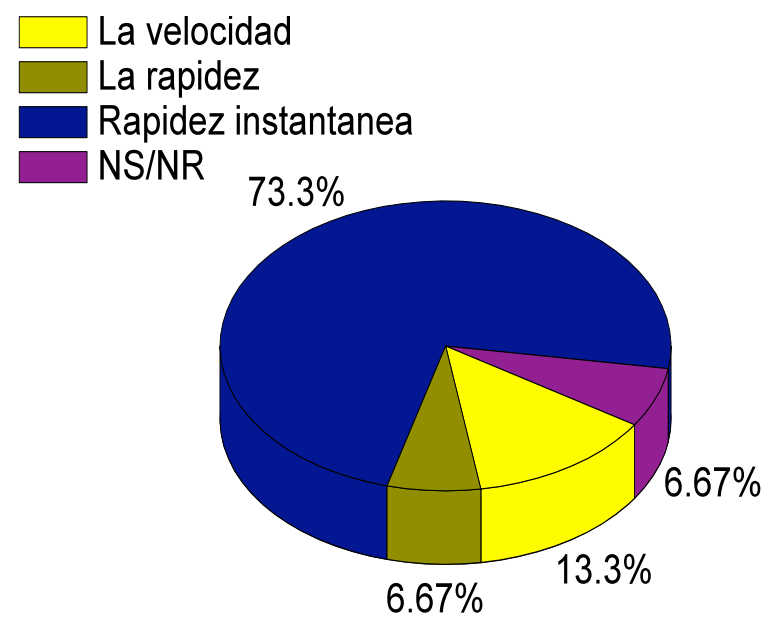

Después

A la tercera pregunta: ¿Cuantas componentes vectoriales tiene la velocidad?, ¿y la rapidez? Respondieron:

Velocidad varias, rapidez mas de una

las componentes son las mismas (distancia y tiem

velocidad varias, rapidez no tiene

NS/NR

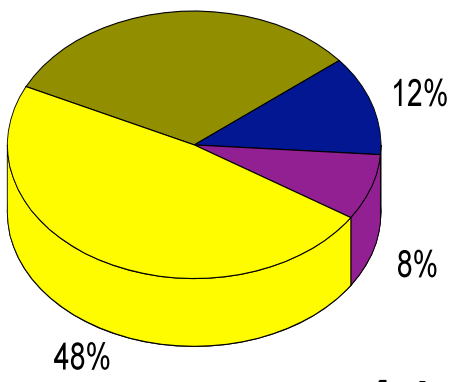

Velocidad varias, rapidez mas de una las componentes son las mismas (distancia y velocidad varias, rapidez no tiene

NS/NR

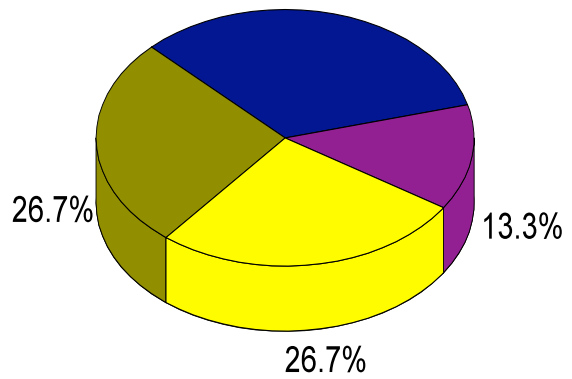

Después

A la cuarta pregunta: De acuerdo a lo observado en la simulación 1 ¿Cual de las esferas ha recorrido una mayor distancia? ¿Por qué? 


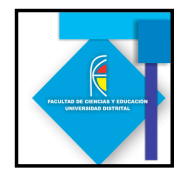

Antes

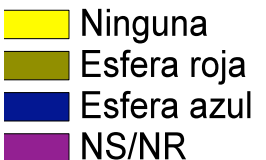

A la quinta pregunta: De acuerdo a la simulación 2 ¿Cuál de las dos esferas tiene más rapidez? ¿Por qué?

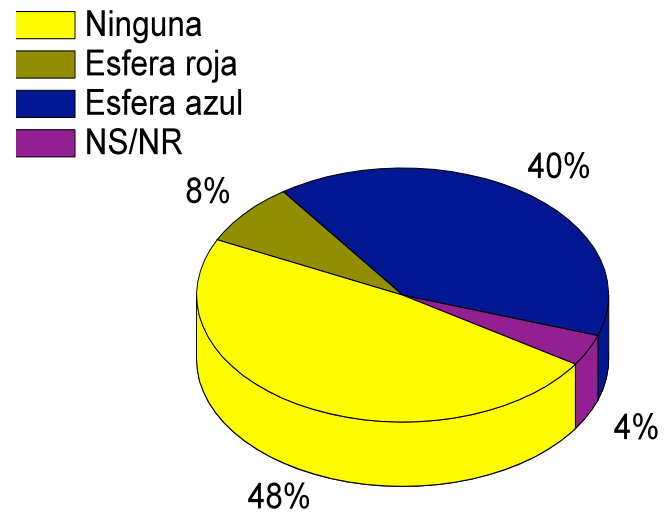

Antes

\begin{tabular}{l}
\hline Ninguna \\
$\square$ Esfera roja \\
Esfera azul \\
NS/NR
\end{tabular}

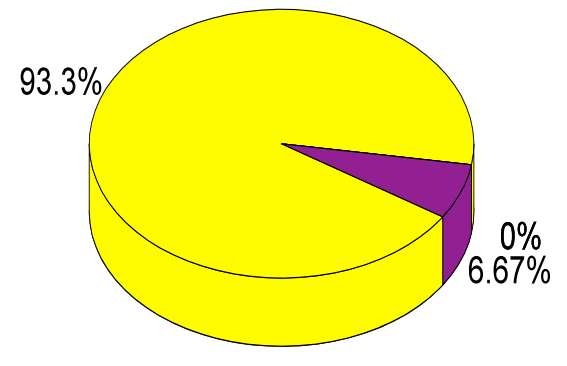

\section{Después}

$8 \%$

$8 \%$

$4 \%$

Después

\section{Análisis de resultados}

Se puede observar una mejoría (aunque no notable) en los conceptos desarrollados por los estudiantes dado que a través del dialogo y los cuestionamientos realizados se pudo llegar a unos modelos conceptuales mas concretos sobre los fenómenos cinemáticos especificados, sobre todo cuando se les preguntaba sobre un caso real donde se puede ver el fenómeno, dado que ellos razonaban de acuerdo a lo ya conocido, a lo confrontado y a un caso especifico observado en su experiencia personal. Aún quedan algunas dudas respecto a las componentes que puede tener el vector velocidad, tal como se puede ver 


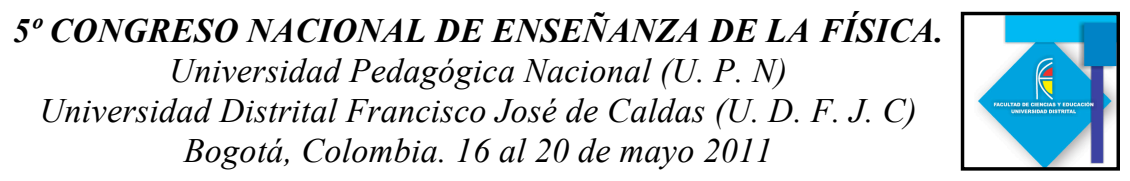

en la pregunta número 3 ya que tiende a confundirse el término componentes con el termino variables, es así como se argumentaba por parte de los alumnos que las

componentes de la velocidad eran distancia y tiempo, y de la rapidez también, eso debería llevar a un estudio profundo a futuro para poder detectar de donde surge este tipo de preconcepciones; a su vez también vale la pena mencionar que pese a los resultados obtenidos al ser tan corto el tiempo de elaboración puede considerarse que fue un preámbulo efectivo para iniciar investigaciones posteriores.

\section{Conclusiones}

Realizando este trabajo se puede concluir que muchas de las estructuras mentales que los estudiantes conciben desde bachillerato no les permiten ser fácilmente encaminados hacia un modelo adecuado conceptualmente, es por esto que se requiere un compromiso por parte de los docentes para elaborar un contenido sobre la asignatura adecuado a los contenidos preconcebidos de los estudiantes acorde al modelo pedagógico con el que estos llegan en su gran mayoría de bachillerato para fundamentar unos conceptos mas firmes. A su vez se pudo observar que la confrontación y el acople entre la clase tradicional, los cuestionamientos, la simulación y el traslado de estos contextos a fenómenos reales se pueden emplear para ayudar a los estudiantes a entender un poco mejor su entorno, además del hecho de generar preguntas como eje fundamental para lograr un espíritu mas investigativo.

\section{Referencias Bibliograficas}

Hurtado, A y Lombana, C. (2006) Experimento y simulación: Opciones didácticas en la enseñanza-aprendizaje de la física. 1. Ed. Bogotá: Universidad Distrital Francisco José De Caldas, 202 p.

Esquembre, F. (2005) Creación de simulaciones interactivas en Java: Aplicación a la enseñanza de la física. 1. Ed. Madrid: Pearson Prentice Hall, 352 p.

Sierra. J L (2002) Estudio de la influencia de un entorno de simulación por ordenador en el aprendizaje por investigación de la física en bachillerato. Madrid: Centro de investigación y documentación científica. p.86 